\title{
Feasibility of Coordinate Measuring System Based on Wire Driven Robot
}

\author{
Ji-Hui Zhou, Qing-Song Cao, Fa-Xiong Sun, and Lan Bi \\ College of Mechanical and Electrical Engineering, East China Jiaotong University, \\ Nanchang 330013, China
}

\begin{abstract}
The coordinate measuring machine systems (CMMS) are widely used for part inspections in manufacturing plants, which mostly include bridge CMMS and horizontal CMMS driven by motor and portable CMMS operated manually. Wire-driven parallel kinematic manipulators (PKMs) have advantages of flexibility, accuracy and large workspace. This paper investigates the feasibility of the coordinate measuring system based on wire-driven PKM. The forward and inverse position solutions are obtained by MATLAB simulation through the kinematic analysis of the manipulator. The controllable workspace with tension conditions and stiffness conditions are obtained by means of the static analysis. Analysis results show that the manipulator have high precision, high speed and large workspace, these advantages just meet the measure requirements of coordinate measuring systems. Finally, a primary experiment is presented to show that the measuring platform of this manipulator can move smoothly and its measurement error is relatively low. The feasibility of taking the wire-driven PKM as coordinate measuring system has been validated by theoretical and experimental study. This research maybe lays a foundation for the further application of wire-driven PKM in the field of coordinate measuring.
\end{abstract}

Keywords: Wire-driven parallel kinematic manipulators (PKMs), Coordinate measuring system, Feasibility.

\section{Introduction}

As one of the most indispensable metrological instruments for inspections and quality control in manufacturing plants, coordinate measuring machine systems (CMMS) are widely used in many fields such as mechanical manufacturing, automotive industry, electronic industry, aerospace industry and national defense industry. The first CMM was developed by the Ferranti Company of Scotland in the 1950s, although this machine only had 2 axes. The first 3-axis models began appearing in the 1960s and computer control debuted in the early 1970s. After the 1970s, CMMS have made rapid development [1]. With the development of modern industry, fast and accurate measurement is an important research subject [2]. At the same time, novel type mechanisms need to be designed.

Wire-driven parallel kinematic manipulators (PKMs) possess a number of promising advantages over the conventional rigid-link manipulators, such as simple and lightweight mechanical structure, high-loading capacity, large workspace, low moment 
inertia and high speed motion. The early study on wire-driven PKMs is the application in cargo handling. Over the years, a number of different wire-driven PKMs designed for a wide variety of applications in large-scale manufacturing such as welding, cutting, grinding, assembly, fixturing, paint stripping, and machining. An example of wire driven robots that are currently in use is the NIST Robot Crane[3]. The NIST Robot Crane is a large-workspace robot for painting and maintaining aircraft which is also suitable for material handling in warehouses and storage facilities [4]. Recently, six degrees of freedom wire-driven PKMs in the application of a large radio telescope is presented [5]. The potential for cable robots are given to be used in a variety of applications, the studies on the wire-driven PKMs will be promoted.

The novel three degrees of freedom (DOF) coordinate measuring machine driven by four wires is proposed in this paper. Based on the kinematic analysis of the manipulator, it is confirmed that this manipulator have large workspace, high moment precision and low moment error after the simulation of MATLAB. A primary experiment is presented to show that the moving platform of this manipulator can move smoothly, its measurement error is relatively low and measurement precision is relatively high. The feasibility of wire-driven PKM used as coordinate measuring system is verified by theoretical analysis and experiment. This study will play an important role in pushing forward the application of wire-driven PKM in coordinate measuring field.

\section{Overal Design}

The basic function of CMMS is to gain the actual shape of a work piece. The actual shape of the work piece is obtained by probing the surface of the work piece at discrete measuring points which can then be analyzed via regression algorithms for the work piece of features. Every measuring point is expressed in terms of its measured coordinates. Based on this principle of measurement, the overall plan design of the measuring system is presented in figure 1 .

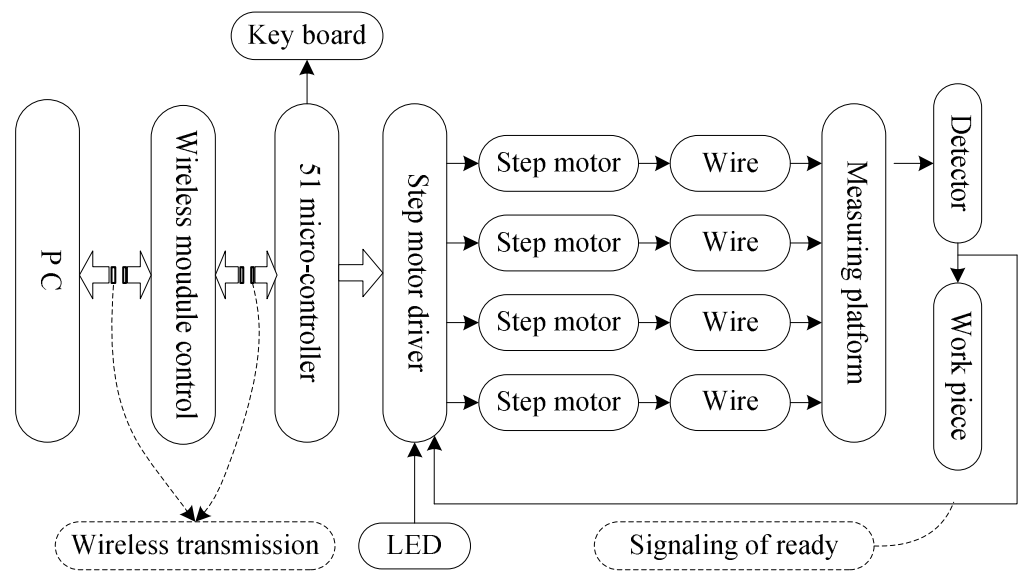

Fig. 1. Block diagram of the system 
The measuring system includes four main components: the main structure, measuring system, touch trigger probe and electrical control system. The main structure is an $1160 \mathrm{~mm} \times 550 \mathrm{~mm} \times 1000 \mathrm{~mm}$ PKMs driven by four wires. The accurate control of the measuring platform is obtained through using step motor to control the lengths of the wires. The step motor controlled by pulse-to-step is generated by a 51 microcontroller. As one of the most important part, the touch trigger probe greatly influences measurement accuracy and precision. The touch trigger probe named TP60 is used in this paper which has a spring loaded ruby ball stylus. The different functions can be realized by the electrical control system through the way of microcontroller programming. The control signal can be import from keyboard or from 51 microcontroller and PC serial communications. After receiving the signal, the microcontroller jump to the corresponding procedure to realize the different functions, such as display coordinates on the LED nixie tube, the measuring platform is programmed to moving up and right. As the probe touched the surface of the component, the stylus deflected and simultaneously the microcontroller sent information of coordinate (X.Y.Z) to the PC computer. Measuring element of the target such as the shape of the component, the shape of the component and position was obtained by fitting the information of coordinate (X.Y.Z).

\section{Theories Analysis}

\subsection{Structure Analysis}

The schematic diagram of completely restrained wire-driven PKM is shown in figure 2.

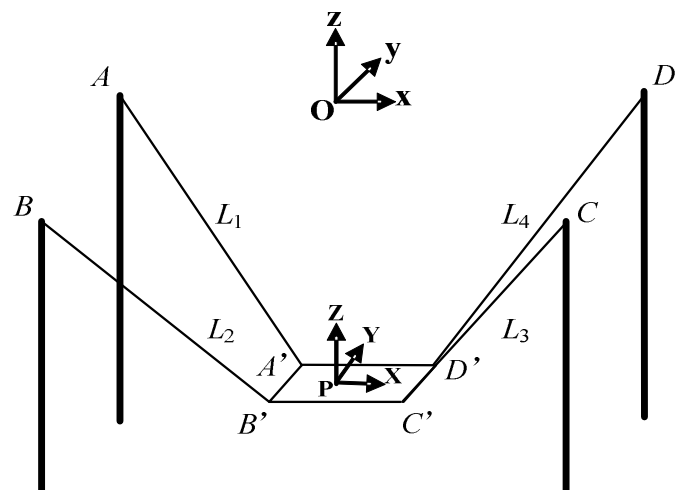

Fig. 2. PKM driven of four wires

The measuring platform is connected to static platform through four driving cables, $A A^{\prime}, B B^{\prime}, C C^{\prime}, D D^{\prime}$. The length of each cable is denoted as $L_{i}(\mathrm{i}=1,2,3,4)$. The cable is connected to the static platform at point $A, B, C$, and $D$. The cable is connected to the measuring platform at point $A^{\prime}, B^{\prime}, C^{\prime}$ and $D^{\prime}$. The center of $A B C D$ and $A^{\prime} B^{\prime} C^{\prime} D^{\prime}$ expressed in $O$ and $P$. Make coordinate system $O$-xyz become the static coordinate system. Let coordinate system $O$-xyz becomes the moving coordinate 
system. The unit vector in the direction of the cable is $e_{i}$. The manipulator has only 3 Cartesian degrees-of-freedom $(x, y$, and $z$ ). In Figure 2, the design parameters for the PKM driven by 4 wires are $2 a$ (static platform length), $2 b$ (static platform width), $2 c$ (measuring platform length), and $2 \mathrm{~d}$ (measuring platform width). $t_{i}$ is the cable tension applied for the cable. $r_{i}$ is the position vector from the origin of $P$ to the cable connection. For static equilibrium the sum of external forces and moments exerted on the measuring platform by the cables must equal to the resultant external wrench exerted on the environment.

$$
F=A T
$$

Where $T=\left(t_{1} \cdots t_{4}\right)$ is the vector of scalar cable forces, $F$ is the resultant external wrench vector exerted on the environment by the measuring platform, $A=\left[e_{1} \cdots e_{4}\right]$ is the statics jacobian matrix.

$L=\left(L_{1} \times e_{1} \cdots L_{4} \times e_{4}\right)^{T}$ is the vector of cable length. $V_{i}$ is the cable translational velocity applied for the cable, expressed in $V . A^{T}$ is the transpose matrix of A matrix. By means of the method of the vector analysis, the following matrix form can be obtained

$$
L=A^{T} V
$$

The analytical expression of cable forces is obtained via the first equation. In other words, to invert (1) we adapt the well-known particular and homogeneous solution

$$
T=A^{+} F+\left(E-A^{+} A\right) q
$$

Where $A^{+}$is the Moore-Penrose pseudo inverse of $A, A^{+} F$ is the minimum norm solution of (1), $q$ is an arbitrary 4 -vector, $E$ is the $4 \times 4$ identity matrix.

\subsection{Position Analysis}

\subsubsection{Inverse Position Analysis}

In static coordinate system, $A^{\prime} B^{\prime} C^{\prime} D^{\prime}$ is expressed in $K_{5} K_{6} K_{7} K_{8}$, while in moving coordinate system, it is expressed in $K_{5}^{\prime} K_{6}^{\prime} K_{7}^{\prime} K_{8}^{\prime}$. The coordinates $\left(X_{p}, Y_{p}, Z_{p}\right)$ is the position of $P$ relating the static coordinate system. According to the closed vector approach, the following equation can be described

$$
K_{i}=K_{i}^{\prime}+P
$$

Therefore, the length of each cable can be calculated as follows

$$
\begin{aligned}
& L_{1}=K_{1}-K_{5}=\left[\begin{array}{c}
c-a \\
b-d \\
0
\end{array}\right]-\left[\begin{array}{c}
X_{p} \\
Y_{p} \\
Z_{p}
\end{array}\right], L_{2}=K_{2}-K_{6}=\left[\begin{array}{c}
c-a \\
d-b \\
0
\end{array}\right]-\left[\begin{array}{c}
X_{p} \\
Y_{p} \\
Z_{p}
\end{array}\right] \\
& L_{3}=K_{3}-K_{7}=\left[\begin{array}{c}
a-c \\
d-b \\
0
\end{array}\right]-\left[\begin{array}{c}
X_{p} \\
Y_{p} \\
Z_{p}
\end{array}\right], L_{1}=K_{4}-K_{8}=\left[\begin{array}{c}
a-c \\
b-d \\
0
\end{array}\right]-\left[\begin{array}{c}
X_{p} \\
Y_{p} \\
Z_{p}
\end{array}\right]
\end{aligned}
$$


The inverse position solutions can be expressed as

$$
\left\{\begin{array}{l}
L_{1}=\left\|L_{1}\right\|=\sqrt{\left(c-a-X_{p}\right)^{2}+\left(b-d-Y_{p}\right)^{2}+Z_{p}^{2}} \\
L_{2}=\left\|L_{2}\right\|=\sqrt{\left(c-a-X_{p}\right)^{2}+\left(d-b-Y_{p}\right)^{2}+Z_{p}^{2}} \\
L_{3}=\left\|L_{3}\right\|=\sqrt{\left(a-c-X_{p}\right)^{2}+\left(d-b-Y_{p}\right)^{2}+Z_{p}^{2}} \\
L_{4}=\left\|L_{4}\right\|=\sqrt{\left(a-c-X_{p}\right)^{2}+\left(b-d-Y_{p}\right)^{2}+Z_{p}^{2}}
\end{array}\right.
$$

\subsubsection{Forward Position Analysis}

The relationship between the length of each cable and $\left(X_{p}, Y_{p}, Z_{p}\right)$ can be obtained via invert equation $L_{i}=\left\|L_{i}\right\|$, so the forward position solutions can be expressed as

$$
\left\{\begin{array}{l}
X_{p}=\frac{L_{1}^{2}-L_{4}^{2}}{4 \times(a-c)} \\
Y_{p}=\frac{L_{2}^{2}-L_{1}^{2}}{4 \times(b-d)} \\
Z_{p}=-\sqrt{L_{4}^{2}-\left(a-c-X_{p}\right)^{2}-\left(b-d-Y_{p}\right)^{2}} \\
L_{1}^{2}-L_{4}^{2}=L_{2}^{2}-L_{3}^{2}
\end{array}\right.
$$

Whatever the position and pose of the mobile platform is, the length of each cable must meet the Eq. (6). The position and pose of the measuring platform corresponding to certain cable lengths thus are determined via the forward position analysis of parallel wire-driven robot.

\subsection{Workspace Analysis}

\subsubsection{Controllable Workspace}

The controllable workspace is defined as a set of position and orientation for the platform, which meet equilibrium of force and torque, and the tension of each cable is required to be positive [6]. The controllable workspace of the manipulators can be determined by checking the tension of each cable on the basis of the principle of vector closure. Therefore, the manipulators to meet the principle of vector closure should be proved firstly.

According to the principle of vector closure, in a 3-dimensional real space, the arbitrary vector $v$ is closed only if vector $v$ has at least 4 vectors $\left(w_{1}, w_{2}, w_{3}\right.$ and $\left.w_{4}\right)$ satisfying the following two conditions. Condition 1 is that in the four vector $\left(w_{1} \ldots w_{4}\right)$, any three vectors are linearly independent; Condition 2 is that $\sum_{i=1}^{4} \alpha_{i} w_{i}=0$ (for any value of i should meet $\alpha_{i}>0$ ).

Let unit vector $e_{i}$ expressed in $w_{i}$. Take the cable tension $t_{i}$ expressed in $\alpha_{i}$. Assume the resultant external wrench exerted on the environment is zero ( the gravity of each rope is ignored), the following matrix can be expressed: $A T=0$. Therefore, 
$\sum_{i=1}^{4} \alpha_{i} w_{i}=0$ can be obtained through the equation of $\sum_{i=1}^{4} t_{i} e_{i}=0$. Because the mechanisms does not produce singular, in the four vector $\left(w_{1} \ldots w_{4}\right)$, any three vectors are linearly independent. The manipulators to meet the principle of vector closure can be proved through the above analysis.

According to the equation of $T=A^{+} F+\left(E-A^{+} A\right) q$, if the tension solution in the above equation can always be made positive for all positive homogeneous solutions regardless of the external disturbance $F$, the boundary of the workspace can therefore be generated by letting $F=0$. Therefore, the tension of each cable can be positive if each element of $\left(E-A^{+} A\right) q$ is positive. So the controllable workspace bound can be expressed as follow.

$$
\left(E-A^{+} A\right)>0
$$

\subsubsection{Workspace with Tension Conditions}

The workspace with tension conditions is defined as a set of position and orientation under the conditions of the controllable workspace in which the tensions $t_{i}$ in the cables must range between a pre-tension $t_{\min }$ and a maximum tension $t_{\max }$ and the gravity or elasticity of each rope is ignored. Therefore, the workspace with tension conditions can be determined by the tensions in each cable and the external forces and torques exerted on the environment. Assume the external forces and torques exerted on the environment is zero, in other words $F=0$. With $k=t_{\max } / t_{\min }$ is defined as critical tension factor, the workspace with tension conditions can be calculated as follows

$$
\left\{\begin{array}{l}
\frac{\max _{i=1 \ldots 4} B_{i}}{\min _{i=1 \ldots 4} B_{i}} \leq K \\
B=\left(E-A^{+} A\right)>0
\end{array}\right.
$$

Where $B=\left(E-A^{+} A\right)>0$ is the $4 \times 4$ matrix.

\subsubsection{Workspace with Stiffiness Conditions}

The workspace with tension conditions for $n$ DOF PKMs is defined as a set of position and orientation under the conditions of the controllable workspace in which the pose greater than $\mathrm{n}$ positive eigenvalues of stiffness matrix is not less than stiffness coefficients $K^{\prime}$ [6]. Therefore, the stiffness matrix should be calculated firstly.

The stiffness matrix of driven by $m(m \geq n+1)$ wires is obtained by Verhoeven [7].

$$
K=K^{0} A \Lambda A^{T}
$$

Where $K^{0}=k_{i} \times L_{i}^{0}$ is the per unit cable length stiffness, $k_{i}$ is the cable stiffness applied to the cable, $L_{i}^{0}$ is the original cable length applied to the cable, matrix A must meet the condition of $F=A T$, with $A^{T}$ is the transposed matrix of matrix $A$, $\Lambda=\operatorname{diag}\left(L_{i}\left(1+K^{0^{-1}} t_{i}\right)\right)$ is a diagonal matrix. The position and pose of the mobile 
platform can corresponding to certain cable lengths and tension, $L_{i}$ and $t_{i}$ are the certain cable lengths and tension.

Generally, $K^{0^{-1}} t_{i} \infty$ is small enough which can be ignored [8]. The manipulators rigidity performance is determined by geometrical arrangement of wires and the position and orientation of measuring platform. According to formula (9) we can conclude that $K$ is a symmetric positive semidefinite matrix which have n positive eigenvalues $\lambda_{K_{i}}(i=12 \cdots n)$ definitely. Therefore, the workspace with tension conditions must satisfy the following constraints

$$
\left\{\begin{array}{l}
\max _{i=1 \ldots 4} \lambda_{K_{i}} \geq K^{\prime} \\
\left(E-A^{+} A\right)>0
\end{array}\right.
$$

\subsection{Analysis of Kinematic Error}

\subsubsection{Analysis of Error Motion}

The kinematic error for measuring platform moving from one place to another can be obtained via plus the error in each coordinate direction respectively. This article scatters a specific space into different points, and uses the kinematics precision analysis in calculating the variation of the position and pose of measuring platform corresponding to allowable pulse-to-step in different point.

Stepper motor can transform the digital input pulse revolving or the straight line increase activity electromagnetism functional element. When stepping drive to receive a pulse, it drives stepper motor rotate in the direction set by a fixed point of view, then the cable length can be changed in a definite value $\Delta L$. The perimeter of the roller for step motor is $60 \mathrm{~mm}$. So $\Delta L$ can be calculated as

$$
\Delta L=\frac{1.8}{360 \times S N} \times 60=\frac{0.3}{S N} \mathrm{~mm}
$$

Among them, $S N$ is the subdivision number of stepper motor. So the minimum change of each cable length is $\Delta L$.

The measuring platform with moving left and right is $X$, moving forward and backward rd is $Y$ and moving up and down is $Z$. When measuring platform moving up and down, 51 microcontrollers give synchronous control of all cable's speed, the cable length can change $\Delta L$ every time. The kinematic error will not produced only in the condition of $x=0$ and $y=0$. When measuring platform moving left and right, 51 microcontrollers give synchronous control of the first cable and the second cable's speed and synchronous control of the third cable and the fourth cable's speed. Note $\Delta L_{1}=m * \Delta L$ is the change of the first and the second cable length, note $\Delta L_{2}=n * \Delta L$ is the change of the third and the fourth cable length. The kinematic error will produced in the condition of $\mathrm{y} \neq 0$. In this paper, $m$ and $n$ are limited to the same ranges, $m, n \in[0,20]$, and let them be the positive integer. The value of $m$ and $n$ can be obtained in the condition of the kinematic error in the $Z$ ordinate direction is the 
minimum count. When measuring platform moving forward and backward, 51 microcontrollers give synchronous control of the first cable and the fourth cable's speed and synchronous control of the second cable and the third cable's speed. Note $\Delta L_{1}=m * \Delta L$ is the change of the first and the fourth cable length, note $\Delta L_{2}=n * \Delta L$ is the change of the second and the third cable length. The method of gain the value of $m$ and $n$ is similar with the analysis of moving left and right.

With different position and pose of the measuring platform, the kinematic error and accuracy will change. So the relationship between the kinematic error, accuracy and some parameters should be analyzed by MATLAB simulation. Using computer simulation, we can conclude that

1) Littler the size of measuring platform, lower the error for all movement and the accuracy for moving up and down, higher the accuracy for moving right, left, forward and backward. Therefore, the size of measuring platform can be made as small as possible but it has a lower limit.

2) The position and pose in the direction of $z$ is the critical element for the accuracy of moving up and down, and littler the count of $z$, higher the accuracy.

3 ) If the error of moving right, left, forward and backward remained to lower, the original position $x, y$ should be positive and remain low around zero, the position $z$ and moving distance $h$ should be remain low.

\subsubsection{The Best Workspace}

A best workspace $B$ which covering $x, y \in(-100,100)$ and $z \in(-924,-724)$ is obtained by computer simulation. In this workspace, the kinematic accuracy in the direction of coordinate $x$ range from $0.0167 \mathrm{~mm}$ to $0.4165 \mathrm{~mm}$; the kinematic accuracy in the direction of coordinate $y$ range from $0.0513 \mathrm{~mm}$ to $0.7244 \mathrm{~mm}$; the kinematic accuracy in the direction of coordinate $z$ range from $0.0105 \mathrm{~mm}$ to $0.0116 \mathrm{~mm}$. Therefore, the kinematic accuracy of measuring platform is relatively high. If the moving distance is $100 \mathrm{~mm}$, the kinematic error in the direction of coordinate $x$ range from $0 \mathrm{~mm}$ to $1.82955 \mathrm{~mm}$; the kinematic error in the direction of coordinate $y$ range from $0 \mathrm{~mm}$ to $1.85239 \mathrm{~mm}$; the kinematic error in the direction of coordinate $z$ range from 0.428648 $\mathrm{mm}$ to $7.17298 \mathrm{~mm}$. Therefore, the kinematic error of measuring platform is relatively low. The above analyzed results show that the manipulators have high kinematics precision and low kinematics error in the workspace $B$, these advantages just meet the measure requirements of coordinate measuring systems.

\section{Experiment}

This part attempts to verify the rationality and feasibility of adopting wire-driven PKM as coordinate measuring system through experiments. As some limitations, the designed probe is replaced by some parts in the following experiment. Therefore, checking the probe contacts with the work piece is determined by visual inspection. Besides, the kinematics error of the measuring platform is discussed, it includes the errors of the probe, the distortion of each rope, external conditions and so on. The material object photography of CMM is shown in figure 3 . 


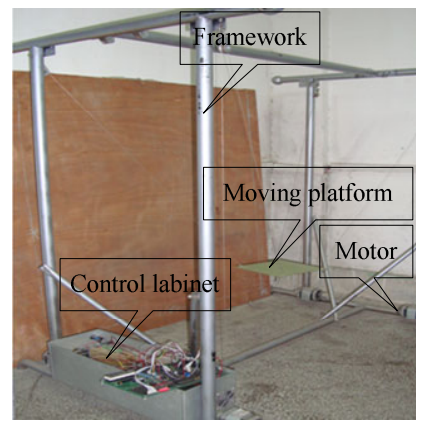

Fig. 3. The material object photography of the designed CMMS

In the experiment, the size of the two rectangular objects is measured in the best working space. The experimental data are in the following table, the error can be obtained via the comparison of measure and actual size.

Table 1. The record of the experimental data (mm)

\begin{tabular}{|c|c|c|c|c|c|c|}
\hline $\begin{array}{c}\text { Initial Coordinate } \\
\text { Values }\end{array}$ & 735.85 & 788.33 & 10.81 & 80.86 & 2.48 & 44.98 \\
\hline $\begin{array}{c}\text { Terminal Coordinate } \\
\text { Values }\end{array}$ & 788.33 & 735.97 & 80.86 & 9.95 & 44.98 & 3.34 \\
\hline $\begin{array}{c}\text { Measure Size } \\
\text { Actual Size }\end{array}$ & 52.48 & 52.36 & 70.05 & 70.91 & 42.50 & 41.64 \\
\hline Error & 3.48 & 3.36 & 6.05 & 6.91 & 6.5 & 7.36 \\
\hline $\begin{array}{c}\text { Initial Coordinate } \\
\text { Values }\end{array}$ & 740.64 & 803.94 & 3.18 & 69.22 & 4.71 & 42.03 \\
\hline $\begin{array}{c}\text { Terminal Coordinate } \\
\text { Values }\end{array}$ & 803.94 & 739.21 & 69.22 & 2.77 & 42.03 & 3.80 \\
\hline $\begin{array}{c}\text { Measure Size } \\
\text { Actual Size }\end{array}$ & 63.3 & 64.73 & 66.04 & 66.45 & 37.32 & 38.23 \\
\hline Error & 3.3 & 4.73 & 6.04 & 6.45 & 5.68 & 4.77 \\
\hline
\end{tabular}

Experimental data show that the measurement error of the designed CMMS is up to a few millimeters, which is somewhat of big. The main factors for the measurement error are human factors, such as the visual error in checking the probe contacts with the work piece and reaction time of human. If the designed probe is used in the experiment, the measurement error should be greatly reduced and it would meet the measure requirements of coordinate measuring systems. Through the observation of 
the experiment we can conclude that the measurement platform can moved smoothly even in the condition of fast moving. The feasibility of adopting wire-driven PKM as coordinate measuring system is verified once again.

\section{Conclusion}

The forward and inverse position solutions are firstly obtained by means of the static analysis and the controllable workspace, workspace with tension and stiffness conditions are presented in this paper. Then the kinematics precision and error analysis of the wire-driven PKM is given by MATLAB simulation based on the kinematic analysis of the manipulator. The best workspace in which the manipulators have high kinematics precision and low kinematics error is obtained. These advantages meet the measure requirements of coordinate measuring systems exactly. Therefore, the theories analysis verifies the feasibility of adopting wire-driven PKM as coordinate measuring system. Furthermore, the overall scheme of CMMS based on wire-driven PKM is put forward in details. Finally, a primary experiment is presented to show that the measuring platform of this manipulator can move smoothly and its measurement error is relatively low. The feasibility of adopting wire-driven PKM as coordinate measuring system is verified both by theoretical and experimental study.

\section{References}

1. Liu, Z., Ni, X.: Present state and developing trend of three-coordinate measuring machines. Machinery 42(480), 32-34 (2004)

2. Zhang, G.: The development tendency of coordinate measuring machines. China Mechanical Engineering 11, 222-226 (2000)

3. Edward, A., Roger, B., Nicholas, D.: Summary of Modeling and Simulation for NIST RoboCrane Applications. In: Deneb Internation Simulation Conference and Technology Showcase, Detroit, MI (1997)

4. Goodwin, K.: RoboCrane Construction of Bridges. In: Transformation Research Record, Transformation Research Board, pp. 42-46 (1997)

5. Zheng, Y.Q., Liu, X.W.: Research survey and development tendency of wire-driven parallel manipulators. China Mechanical Engineering 14(9), 808-810 (2003)

6. Verhoeven, R., Hiller, M., Tadoroko, S.: Works-pace, stiffness, singularities and classification of tendon-driven Stewart platforms. In: Proceedings of the 6th International Symposium on Advances in Robot Kinematics, pp. 105-114 (1998)

7. Ming, A., Higuchi, T.: Study on Multiple Degree of Freedom Positioning Mechanisms Using Wires (Part I): Concept, Design and Control. International of the Japan Society for Precision Engineering 29(6), 131-138 (1994)

8. Liu, X.W., Zheng, Y.Q.: Kinematic analysis of a 6-dofwire-driven parallel kinematic manipulator. Chinese Journal of Mechanical Engineering 38(supplement), 16-20 (2002) 